\title{
Single-Event Effects Ground Testing and On-Orbit Rate Prediction Methods: The Past, Present, and Future
}

\author{
Robert A. Reed, Member, IEEE, James Kinnison, Member, IEEE, Jim C. Pickel, Fellow, IEEE, \\ Stephen Buchner, Member, IEEE, Paul W. Marshall, Member, IEEE, Scott Kniffin, Member, IEEE, and \\ Kenneth A. LaBel, Member, IEEE
}

\begin{abstract}
Over the past 27 years, or so, increased concern over single-event effects (SEEs) in spacecraft systems has resulted in research, development, and engineering activities centered around a better understanding of the space radiation environment, SEE predictive methods, ground test protocols, and test facility developments. This research has led to fairly well developed methods for assessing the impact of the space radiation environment on systems that contain SEE sensitive devices and the development of mitigation strategies either at the system or device level. However, as new technology has emerged, these ground test and predictive methods have certain short falls.
\end{abstract}

Index Terms-Radiation effects in microelectronics, rate predictions, single-event effects (SEEs), space radiation, test facilities.

\section{INTRODUCTION}

A S AN ION passes through a semiconductor it generates electron-hole pairs; this process is known as direct ionization. The charges either recombine or propagate through a semiconductor via drift or diffusion. Because the function of active microelectronic or photonic component is governed by the controlled injection of charge into the depletion layers of $\mathrm{p}-\mathrm{n}$ junctions, the uncontrolled charge injection resulting from ionization can produce an array of effects on the device operation. These effects are known as single-event effects (SEEs).

SEE ground-based testing is done to characterize how a microelectronic device responds to a single particle (neutron, proton, or other heavy ion) interaction with the atoms that makeup the semiconductor. On-orbit rate predictions methods have been developed that use the ground test characterization along with the space radiation environment definition to estimate the frequency of occurrence for a specific SEE.

There is a long list of various types of SEEs (sometimes called the "single-event alphabet soup"). The most studied are

Manuscript received March 27, 2003. This work was supported in part by the NASA NEPP/ERC Project and the Defense Threat Reduction Agency.

R. A. Reed and K. A. LaBel are with the NASA Goddard Space Flight Center, Greenbelt, MD 20771 USA.

J. Kinnison is with JHU/Applied Physics Lab, Laurel, MD 20723 USA.

J. C. Pickel is with PR\&T, Inc., Fallbrook, CA 92028 USA

S. Buchner is a NASA/GSFC support contractor with QSS Inc., Seabrook, MD 20706 USA.

P. W. Marshall, is with NASA Goddard Space Flight Center, Greenbelt, MD 20771 USA.

S. Kniffin is a NASA/GSFC support contractor with Raytheon ITSS, Greenbelt, MD 20770 USA.

Digital Object Identifier 10.1109/TNS.2003.813331 single-event upset (SEU), single-event latchup (SEL), singleevent gate rupture (SEGR), and single-event transients (SET). Another paper, by Dodd [1], in this Special Issue provides a review of the physical mechanisms for SEEs and gives the definition of most SEEs that occur in modern technologies, including those listed above.

In April of 1996, several authors published an IEEE TRANSACTIONS ON NUClEAR SCIENCE (TNS) Special Issue on "Single-Event Effects and the Space Radiation Environment." That Special Issue covered topics that includes SEE rate predictions approaches, test facilities available at the time, test issues for various technologies, as well as the components of the space radiation environment that must be considered when evaluating SEEs in a device.

In this paper, we summarize the concerns and issues for modern devices by providing a historical account of the early days of SEE testing and space observation, an overview of the traditional assumptions used to develop SEE test approaches, a listing of the SEE test facilities available today, a review of SEE rate prediction approaches, and finally a listing of some of the observed phenomena that serve as a reminder that the traditional methods may not be applicable to all modern day technologies.

The works referenced in this paper-and many others that have been published in the IEEE TNS, the Proceedings of the Radiations Effects in Components and Systems (RADECS) Conference, the IEEE Nuclear and Space Radiation Effects Conference's Radiation Effects Data Workshop record, and RADECS Radiation Effects Workshop-present testing methodologies and rate prediction techniques that deal with the issues raised here and that are successful in providing the data needed to develop event rate estimates for space application design.

\section{SEES CIRCA 1975-1980}

Research on SEEs in microcircuits began as most radiation effects research does; in 1975 an anomaly occurred on a earth orbiting spacecraft that could not readily be explained by from known phenomenon. (The possibility of cosmic ray induced SEU in microcircuits was predicted by Wallmark et al. [2] in 1962.) The first work detailing this new phenomenon was published in a paper in 1975 by Binder et al. of the Hughes Corporation [3]. We quote them here: 
"Anomalies in communication satellite operation have been caused by unexpected triggering of digital circuits ... The purpose of this paper is to investigate interactions with galactic cosmic rays as an additional mechanism."

The authors were able to show that the anomaly was due to SEUs in a digital flip-flop circuit. They developed a rate prediction approach based on device and transistor parameters, charge collection efficiency, and the solid angle and energy spectrum of impinging cosmic rays. The calculation was within a factor of two of the observed rate. While this work marked the start of a new era of radiation effects, the radiation effects community largely overlooked it.

A second paper, motivated by yet another spacecraft anomaly, by Pickel and Blandford [4], was published in 1978 that details the development of a heavy ion-induced rate prediction model that utilizes the concept of describing the space radiation environment as an LET distribution, or Heinrich distribution [5]. They compared their calculation of the SEU rate for a NMOS dynamic RAM to on-orbit results and found agreement to within a factor of two. The observed error rate for the system was near one error per day, a significant enough result to catch the attention of many of the radiation effects experts of that time.

Another paper published in 1979 by May and Woods [6] detailed the first reported alpha particle induced SEUs. The alpha particles were emitted from trace amounts of uranium and thorium present in the packaging materials.

Also in 1979, two independent research groups uncovered the fact that the recoil products from a proton-induced nuclear spallation reaction could have sufficient LET to cause an SEU. Wyatt et al. reported on ground test results on two types of $4 \mathrm{k}$ DRAMs [7]. They observed upsets occurring for protons energies ranging from 18 to $130 \mathrm{MeV}$. At the same time Guenzer et al. [8] studied and reported on proton and neutron-induced effects. They observed SEUs in 16k DRAMs for neutron energies that ranged from 6.5 to $14 \mathrm{MeV}$ and for $35 \mathrm{MeV}$ protons.

There are very distinct differences in SEE testing and rate prediction approaches between SEEs induced by direct ionization from the primary particle and indirect ionization by reaction products from a nuclear collision. Typically, effects are dominated by direct ionization for ions with $Z>1$ (known in the radiation effects community as "heavy ions"). For neutrons and protons the effects are typically dominated by indirect ionization. Because of the different mechanism involved, the methods used to determine rate predictions - and thereby different test methods - are very different.

SEUs were not the only topic of discussion in the early days of SEE. The discovery of heavy ion-induced SEL was first published in 1979 by Kolasinski et al. [9]. The authors reported on SEL induced in SRAMs by heavy ion nuclei.

By 1980, the combined impact of all of these papers was significant enough to motivate researchers, project managers, and design engineers to pay attention to this new phenomenon of radiation-induced effects. Shortly after these early papers were published, a sequence of symposia devoted to SEEs was held. This meeting-The Single Event Effects Symposium, today a biennial event - was (and still is) critical to the development of the current understanding of SEE.
It is interesting to note that the early work detailing the analysis and rate prediction approaches have proven to be very robust even when applied to most modern day technologies. However, there are certain cases where these analyses fail to predict the device response.

\section{TRaditional Assumptions USED to DeVElop SEE TEST APPROACHES}

\section{A. Introduction}

In this section, we discuss some of the assumptions and methods used to perform SEE testing (Section VI describes cases where these fundamental assumptions are shown to be inadequate.) First, we summarize the space environment, then we discuss proton and heavy ion SEE ground testing, and finally we discuss system level testing implications.

\section{B. SEE Space Environment}

The space charged-particle environment responsible for SEEs is dominated in particle count by energetic protons, with smaller contribution from heavier ions $(Z>1)$. However, various sources generate these particles, and the characteristics of the environment vary in distinct regions of space. The environment is traditionally divided into three parts-galactic cosmic rays, particles from solar events, and particles trapped in planetary magnetospheres.

Galactic cosmic rays (GCRs) are highly energetic ions $(Z \geq$ 1 ) that arise from sources outside the solar system and are generally considered to form a spatially constant background modulated by the changes in the solar magnetic field during the solar cycle and by the presence of planetary magnetospheres.

Solar events produce a range of energetic ions, but the maximum energy of these particles is much lower than for GCRs. Correspondingly, the solar ion environment is significantly modified by spacecraft shielding, by planetary magnetic fields, and by phasing within the solar cycle. Also, solar events are of short duration, so the solar ion environment consists of a sequence of impulsive bursts of ions that can dramatically raise the SEE rate for a short time.

Charged particles can be trapped in planetary magnetospheres, and spacecraft in these fields will experience SEEs at rates that strongly depend on the details of the orbit. The most important example of this environment is the Earth's Van Allen belts; all low Earth orbiting spacecraft must take into account the presence of the trapped proton belt, including deviations from a dipole model such as the South Atlantic Anomaly (SAA). Another important example is the environment of ions such as sulfur trapped in the Jovian magentosphere.

The space environment is modified by shielding associated with the structure of the spacecraft around a device in orbit as well as the packaging of the device itself. While this effect is small for energetic cosmic rays, the spectrum of lower energy ions-such as those produced by solar events or trapped protons - is significantly altered by the presence of material around devices and must be included in rate prediction. Most computer codes used to estimate environments include transport of ions through an assumed thickness of material before calculating the spectrum used for rate estimation. 
There have been several Nuclear and Space Radiation Effects Short Courses that give a detailed description of the charged particle environment [10]-[13]. Also Barth's paper in this Special Issue [14] gives a good review of the space radiation environment.

\section{Device Level Testing}

1) Heavy Ion SEE Testing: The event rate for a given effect in space is determined by a combination of environment and device characteristics, which are assumed to be completely described by the geometry of a sensitive volume and a critical charge associated with the effect in question for a given cell within a device. In most cases, a device is modeled as an array of identical thin right-rectangular parallelepiped sensitive volumes-we discuss rate prediction approaches later in this paper. Device level SEE testing helps to define some of the critical parameters that are used to determine the on-orbit event rate.

The fundamental assumption associated with heavy ion SEE testing is that the cross section only depends on the "effective LET" of the incident particle, that is, the nominal LET of the particle divided by the cosine of the incident angle, where the angle is that from the normal to the die surface. Division by cosine comes from the fact that the pathlength of the ion through the sensitive volume increases with the angle of incidence. This increased pathlength gives rise to more charge being generated in the sensitive volume.

The end result of an SEE test is a measure of the cross section as a function of effective LET. The cross section usually takes the form of a curve with onset of SEE at some threshold LET which then rises to an asymptotic value at higher LET. The critical charge is determined from the threshold LET, while the asymptotic cross section gives the area of the sensitive volume. When combined with the thickness of the sensitive volume-typically derived from the architecture of the device-the parameters derived from the cross section are sufficient to allow calculation of SEE rates for many technologies in any given space environment where $Z>1$.

A typical test consists of a series of mono-energetic exposures for beams over a range of LETs (or effective LETs). During each exposure, the device is placed under bias, either active or passive. Events of interest are counted for a known incident fluence, and the cross section is given by the ratio of number of events to the effective particle fluence (where the effective fluence is the product of the normal incident fluence and the cosine of the angle-this correction is for the reduced effective exposure area of the die surface). Authors of research and test data reports often omitted the word "effective," even when the heavy ion beam is at some angle relative to the normal to the die.

Some of the early work was devoted to understanding SEUs in static RAMs, which are the best example of many of the assumptions in SEU testing. Each RAM cell is-to first order-identical, and from an SEE perspective, the device is easily seen to be an array of identical sensitive volumes. Tests are usually performed by loading a pattern in the memory array, exposing the device to a known fluence of charged particles at a particular LET. After the exposure, the array is interrogated to count the number of flipped bits, from which

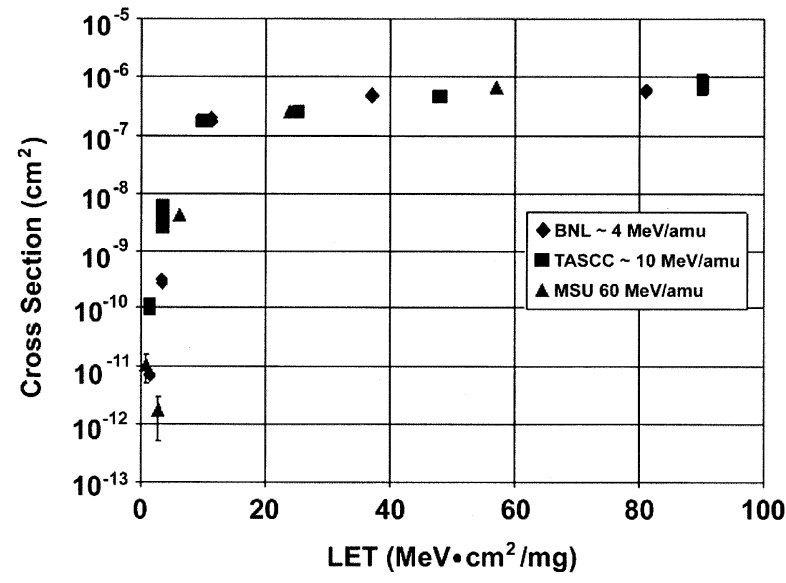

Fig. 1. Example cross-section curves for a Matra $32 \mathrm{~K} \times 8$ SRAM (circa 1998).

the cross section is calculated. Since each sensitive volume is identical, the per-bit cross section is simply the measured cross section normalized by the number of bits in the memory array. A complete experiment uses many LET values to fully map the cross section of interest. Since the number of different beams is limited at a test facility, some method of changing the LET of the beam-often, non-normal angles of incidence or degrader foils to change the beam energy - is used to provide as many data points as needed. Fig. 1 is an example cross-section curve for a Matra $32 \mathrm{~K} \times 8$ SRAM.

The cross section often depends on other factors such as temperature or electrical bias, or deviates from a strict dependence on effective LET. Even in the simplest cases such as SEU in static RAMs, significant deviations from the basic testing assumptions are observed. For example, if the array of identical well-defined sensitive volumes were strictly true, the cross section would be a step function with respect to LET. In reality, the cross section increases with finite slope in the threshold region, followed by a knee region and a more gradual approach to the asymptotic cross section than seen in a step function. These deviations can be due to statistical variations in the sensitive volume geometry or in the critical charge for a volume and are significant for calculating event rates from cross-section data (see Section IV).

Another source of deviation in the shape of the cross section occurs when more than one sensitive volume is found in a cell, or when several different types of cells are present, each with their own characteristic sensitive volumes.

As a final example, experimenters often find discrepancies between cross sections measured at the same effective LET with different beams at different incident angles. These discrepancies have been, in part, attributed to the deviation from the inverse cosine relationship between LET and incident angle. These issues have been studied in detail over the last decade, and in each case, methods for dealing with deviations have been developed [15].

SEU testing can often involve other serious complications and deviations to the basic methodology outlined above. For instance, microprocessors contain many registers and latches that may or may not be active at a given time depending on 
the program running on the microprocessor. Therefore, the device upset cross section strongly depends on the software used during the measurement, and the problem of actually detecting an error becomes quite complex. Early on, Koga et al. developed several different methodologies for testing microprocessor devices [16]. These methods are based on comparison between irradiated devices and golden devices or simulated golden devices and are the basis for much of the microprocessor testing today. Other examples include SEU testing of analog-to-digital converters [17] where the definition of an error is in question and devices such as field programmable gate arrays [18] where inadvertent rearrangement of the circuit design while under irradiation causes complications in the operation of a device. In each example, methods for determining cross sections and event rate estimates have been developed that allow conservative circuit design in space applications.

Effects other than upset also provide complexity beyond the basic SEU test. Latchup sensitivity in a device is a function of LET, but also of operating voltage, temperature, and range of the incident particle in the device. In many devices, care must be taken to use beams with sufficient range to deposit charge in latchup sensitive volumes deep within the device, which calls into question the concept of effective LET for latchup.

Also, in devices susceptible to gate rupture or burnout, the goal of a test is not to define the cross section as a function of LET, but to measure susceptibility for various device parameter settings. These data allow the definition of safe operating regions for the device; when an engineer uses the device in the "safe region" the susceptibility to the effect is eliminated or greatly reduced [19], [20].

2) Proton SEE Testing: Energetic protons generally do not deposit enough energy in a sensitive volume to directly cause SEEs. However, approximately one in $10^{4}$ to $10^{6}$ protons undergo nuclear reactions with the $\mathrm{Si}$ atoms in the device and produce energetic residual nuclei. If produced in or near a sensitive volume, the residual nuclei can deposit enough energy to cause an SEE. The residuals that cause events are short-range ions that deposit most or all their energy within the sensitive volume.

Proton testing proceeds much like heavy ion testing in that the sample is exercised while exposed to a beam for a given fluence. Events are counted in each exposure, and the event cross section is calculated by dividing the number of events by the fluence for the exposure (recall that the effective fluence is used for heavy ion testing). This procedure is repeated over a range of proton energies to fully characterize the cross section as a function of energy. For the most part, exposures are done in-air.

Three issues, however, make proton testing significantly different from heavy ion testing. First, and foremost, samples experience significant total ionizing dose damage when exposed to proton beams, and the event cross section can be different as damage accumulates. Care must be taken to plan experiments so that the device characteristics are not unduly altered during the measurements. Second, since the nuclear interaction probability does not depend on the beam incident angle and the reaction products deposit nearly all their energy in the sensitive volume, the cross section is assumed to only depends on the proton energy. As a result, for proton testing, there is no equivalent concept to the effective LET used in heavy ion testing. Finally, the physical layout of proton exposure facilities and safety concerns for human experimenters adds considerable complication to experiment and equipment design.

\section{Spacecraft System Impact on SEE Testing}

SEEs can lead to changes in the macroscopic behavior of a spaceflight system. For instance, changes in microprocessor code in an SRAM will, at a minimum, cause erroneous execution of the program. In the case of SEUs or SETs in a circuit, the system event rate is generally not the sum of the individual device event rates. Instead, the system event rate is determined by a complex interplay of the location of a given event, the sensitivity of other devices in the circuit, and the timing of the event relative to the activity of the system. If the event rate is low, or if the interaction between devices is simple, the sum of event rates is a reasonable approximation to the system event rate. However, in a few instances, the additive approximation is inadequate, and the system as a whole must be tested.

This testing is generally performed by irradiating devices individually while operating the system. Events observed at the outputs of the system are counted as with a device-level test. In some cases, a device-level test cannot be performed outside the context of a system. For example, a microprocessor test is impractical to perform at the device level. When embedded in a computer system, however, a microprocessor can be readily tested.

Devices sensitive to SEE are often used in space applications. Many mitigation techniques have been developed over the years, from error detection and correction in memories to latchup protection circuits for individual devices in a system. The combination of sensitive device and mitigation is a system that must be tested to verify proper operation, especially when a destructive effect is mitigated. Mitigation validation is performed by irradiating the sensitive device in a system with mitigation.

In order to be effective, system-level testing requires special consideration. The event cross section strongly depends on the details of the system design and function, including any software that might be executed by the system. As a result, the system tests must be as close to the flight system as practical for reliable test results.

From a modern-day spacecraft developer's point of view, the overriding principle is that SEEs are a system concern and must be understood and managed at the system level. This "management" can be as simple as to select a device that does not exhibit a particular SEE or as complex as error correction and detection schemes that produce impact avoidance or system recovery when an SEE occurs. Independent of the methods used, the impact of SEEs on the system must be understood to insure reliable spacecraft operation.

\section{SEE TEST FACILITIES}

\section{A. Introduction}

While each SEE requires special test considerations, all tests share common components. The basic SEE test consists of a sample exposed to a series of mono-energetic beams over a range of heavy ion LETs - or over a range of energies for proton 
tests-while being exercised in some way to look for the effect of interest. Components of any SEE test are: 1) a source of beams with the required characteristics; (2) a beam monitoring and control system; 3 ) a mounting and positioning system to hold the sample in the beam; and (4) a system for biasing the sample and measuring its electrical behavior to detect the SEE. In most facilities of general use in SEE testing, the facility provides the beam, beam monitoring and control, and sample mounting; the user is generally responsible for providing properly prepared samples, an electrical system to bias and monitor the sample, any interface hardware used to adapt the user's system to the facility equipment, and equipment to provide for special test needs such as sample thermal control.

\section{B. Heavy Ion Test Facilities}

There are seven major heavy ion beam facilities-five in the U.S. and three in Europe - that are currently available or will be available in the near future for SEE testing (others facilities exists, but are not considered to be major test facilities at this time). These highly capable SEE test facilities are located at laboratories used for basic physics research; generally, basic research will be higher priority at these laboratories than SEE testing. Part of the challenge in providing SEE test facilities will be to negotiate sufficient test time to meet the needs of the aerospace community while not unduly disrupting basic research at a laboratory. Each of the facilities described here produces a distinct set of beams and is a unique set of compromises between cost, ease of use, and space environment simulation fidelity.

A major consideration for determining the fidelity of a test is the energy of the beam for a given LET; higher energy beams tend to more accurately reproduce the effects of the space environment at the expense of higher cost and greater complexity.

In 1987, the Single Event Upset Test Facility (SEUTF) was built by a consortium of U.S. government agencies and Brookhaven National Laboratory (BNL) in response to increasing demand for SEE data for spacecraft hardware design and qualification. Since becoming operational in 1988, the SEUTF has been available to users including government, academic, and commercial institutions. The SEUTF consists of a test station attached to the east beam line of the Brookhaven Tandem Van de Graaff Facility (TVDGF) and is maintained and supported by the TVDGF [21]. The TVDGF is a low energy accelerator compared to all other test facilities. The maximum energy for the standard beams is on the order of a few $\mathrm{MeV}$ per nucleon. Downstream from the beam TVDGF control and measurement system, a system of five detectors is used to independently monitor the beam just prior to the SEUTF test chamber. Four of the detectors are placed evenly around the edge of the beam to measure fluence during test runs, while the fifth is mechanically inserted into the center of the beam between runs. Since the TVDGF provides low energy beams, experiments must be performed in a vacuum. The SEUTF chamber is a large vacuum chamber attached to the end of the beam-line. The main SEUTF hardware interface is a three-axis goniometer stage driven by absolute-encoded stepper motors. The stage is designed to provide travel in all three linear dimensions as well as revolve about the beamline axis and rotate about the vertical axis of the stage to change the incident angle of the beam relative to the sample surface. The SEUTF is controlled through a custom-designed software package that includes the local user beam control and monitoring as well as control of the sample positioning system and data logging for each run.

The 88-in Cyclotron at Lawrence Berkeley National Laboratory (LBNL) has been used for SEEs testing by experimenters from the Space Science Applications Laboratory (SSAL) at the Aerospace Corporation since 1978, the early days of the field of study [22]. In 1996, the third-generation Single Event Effects Facility (SEEF) became operational as the latest facility for SEE testing at LBNL [23]. The cyclotron can develop beams in cocktails which are mixtures of elements with constant charge-to-mass ratio. Each element will have the same energy per nucleon and so will have different incident LET. Two standard cocktails are available at $4.5\left(1-62 \mathrm{MeV}-\mathrm{cm}^{2} / \mathrm{mg}\right)$ and $10 \mathrm{MeV}$ per nucleon $\left(1-55 \mathrm{MeV}-\mathrm{cm}^{2} / \mathrm{mg}\right)$. Low LET ions can be added to each cocktail to extend the LET range of each below $1 \mathrm{MeV}-\mathrm{cm}^{2} / \mathrm{mg}$. Before passing to the test chamber, beams from the 88 -in Cyclotron are routed through a beam diagnostic system which is used to collimate and shutter the beam, measure beam characteristics before test runs, measure relevant beam parameters during exposures, and allow alignment of samples with the beam. This is accomplished with a set of particle detectors and filters and a mirror for the alignment laser. All of the elements in the beam diagnostic system except the filter wheel are mounted on sliding stages and can be inserted or removed from the beam via software on the SEEF control computer. The main test chamber is a large vacuum enclosure of about $1 \mathrm{~m}^{3}$ volume surrounding a four-axis motion system on which test hardware is mounted. The four-axis motion system provides linear travel across the beam horizontally and vertically, as well as rotation about the beam axis and about the vertical axis normal to the beam (to change the incident angle of the beam with respect to the sample surface).

The Texas A\&M University (TAMU) Cyclotron Institute, jointly funded by the State of Texas and the U.S. Department of Energy, operates a K500 superconducting cyclotron to support research in nuclear physics and chemistry, as well as applied research in space science, materials science, and nuclear medicine. The Cyclotron Institute has established the Radiation Effects Facility (REF) as a permanent test area and has offered it for use by commercial, government and educational organizations to study SEEs in microelectronic and related radiation effects research [24]. The Cyclotron Institute is planning a series of upgrades which will link the previously existing 88-in cyclotron with the K500 cyclotron to expand the available beams and increase the usefulness of the facility; one impact of this upgrade is that more time may be available for SEE testing [25]. A number of high energy beams have been developed by the Cyclotron Institute as "standard" beams for REF. These beams range in energy from 12.5-55 MeV/amu. Many of these beams can be used without a vacuum chamber, which greatly simplifies the test equipment interface. REF provides beam monitoring and control in a manner similar to the systems used at SEUTF and SEEF. Since the beam 
LET can be changed with degrader foils, REF also includes a silicon transmission detector that can be used to characterize the degraded beam. Two systems for hardware interface are available at REF-a target chamber system for lower energy beams and an in-air positioning system for higher energy beams. The target chamber is a cylindrical vacuum chamber 76 $\mathrm{cm}$ in diameter and $76 \mathrm{~cm}$ high. Both chambers have automated motion control. Target position verification is performed with a camera co-aligned with the beam axis and a laser that crosses the beam path in the center of the chamber.

The National Superconducting Cyclotron Laboratory (NSCL) at Michigan State University operates a K500 and a K1200 cyclotron for basic nuclear physics research. Over the last five years, beam time has been available on a limited basis for SEEs testing on the K1200, mainly through researchers at Goddard Space Flight Center. In 1999, construction began on an upgrade project that has significantly upgraded the basic physics research capability of the NSCL. The upgrade was accomplished by combining an electron cyclotron resonance source with the K500 and K1200 cyclotrons linked in series and by replacing the fragment separator with a higher acceptance separator [26]. With this upgrade, NSCL can now produce heavy ion beams for all elements with energy higher than that available at all other U.S. facilities except the Alternating Gradient Synchrotron/Relativistic Heavy Ion Collider at Brookhaven National Laboratory. As part of the NSCL upgrade, NSCL staff and NASA Goddard Space Flight Center staff are preparing a facility at NSCL called the Single Event Effects Test Facility (SEETF). The facility consists of a beamline and target area with associated interface, monitoring and control hardware modeled after the systems found at current test facilities. Beams with energy from 20-200 MeV/amu will be available for ions from deuterium to uranium. The current NSCL plan is to provide $300-600 \mathrm{~h} /$ year of beam time to SEE testing. User interface at the SEETF is under development and has been designed to be similar to existing facilities as much as possible.

Fig. 2 plots typical heavy ion LET versus range values in silicon that are attainable at each of the facilities listed above. At several of these facilities, the LET-range values can be changed by tuning the accelerator to a specific beam energy. Degraders can also be used, but the beam energy straggle and uniformity must be verified. (The data for SEETF are the expected LETrange values.)

Cyclotron Research Centre at Louvain-la-Neuve, Belgium [27], operates three cyclotrons capable of delivering of heavy ions up to $27.5 \mathrm{MeV} / \mathrm{amu}$. The cyclotron has two different cocktails of heavy ions used for SEE testing. The test chamber is a $71 \times 54 \times 76 \mathrm{~cm}^{3}$ vacuum chamber with a multidirectional motion controller for moving the device relative to the ion beam. The dosimetry system is similar to the other heavy ion test facilities.

l'Institut de Physique Nucléaire (IPN) in Orsay, France, operates a Tandem Van de Graaff that has used extensively for SEE testing for more than 15 years [28]. The facility setup is similar to the BNL SEUTF as is the range of ions and energies. Testing is done in a vacuum. Dosimetry is similar to the other low energy SEE test facilities.

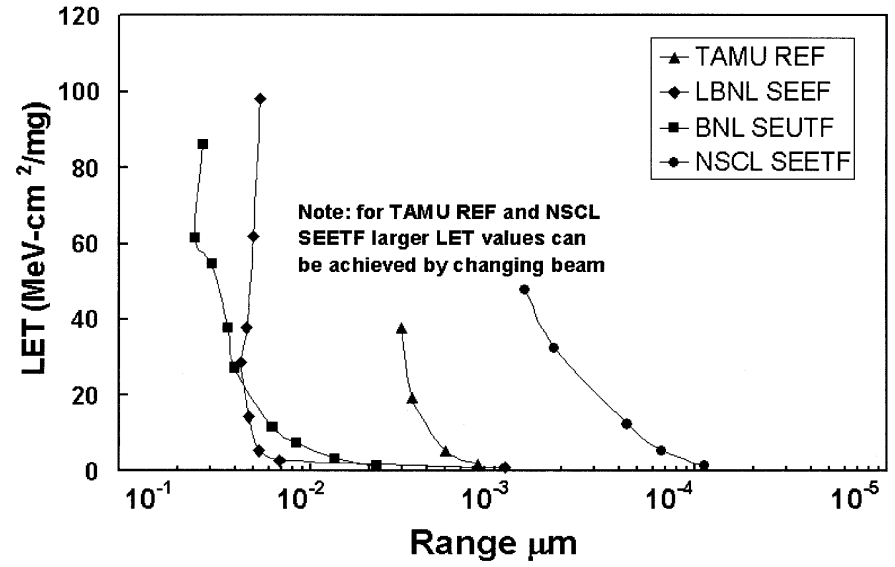

Fig. 2. Typical LET-range values for the facilities listed above. Other values can be achieved by using beam energy tunes or degrading the beam energy.

The Grand Accelerateur National D'Ions Lourds (GANIL) is located in Caan, France [29]. A cyclotron is used to accelerate heavy ions up to $>50 \mathrm{MeV} / \mathrm{amu}$. SEE testing has been performed there for more than seven years.

The Chalk River Laboratories Tandem Accelerator and SuperConducting Cyclotron (TASCC) operated a superconducting cyclotron that was used for SEE testing until the late 1990s. The facility was closed permanently in August of 1997.

\section{Proton Test Facilities}

There are six major proton test facilities; four U.S., one European, and one Canadian (others exists and are used from time to time, but are not considered to be mainstream facilities at this time). These facilities are used for SEE, total ionizing dose, and displacement damage studies. Like the heavy ion SEE facilities these test facilities are located at laboratories used for the most part to carry out basic physics research or cancer therapy-again, this work is a higher priority for these laboratories.

These facilities rely on three primary dosimetry systems to determine the flux and uniformity of the beam: scintillators (usually plastic/organic), secondary electron monitors, and Faraday cups. Additionally, radiochromic films may be used to determine qualitative beam uniformity. All of these facilities have test stands that allow open-air exposures (not in a vacuum). It is widely accepted that the dosimetry at these proton facilities is reasonably accurate, at least within $10 \%$. The next few paragraphs list, in no particular order, the major facilities and gives some information about the facility.

The University of California at Davis's Crocker Nuclear Lab (CNL) [30] has a isochronal cyclotron proton accelerator. It can achieve energies in the 1-68 MeV range. The cyclotron is energy tunable. The beam spot uniformity across the maximum 6 -cm diameter is better than $10 \%$. Beam dosimetry is achieved from calibrated secondary electron emission monitors, these are calibrated to a direct faraday cup measurement.

Indiana University (IU) Cyclotron Facility [31] has a cyclotron/synchrotron (cyclotron only for SEE). The energy peaks at $230 \mathrm{MeV}$ (typical operation is near $200 \mathrm{MeV}$ ) and can be tuned. The beam spot can be up to 7-cm diameter beam spot. 
A second beam line is currently being developed by IU and NASA Johnston Space Center staff. The dosimetry is obtained via a faraday cup and secondary electron emission monitors.

Lawrence Berkeley National Laboratory [23] has an 88-in cyclotron (described above). The proton energy range is $1-55 \mathrm{MeV}$ tuned. The beam spot is $4^{\prime \prime}$ diameter. The dosimetry is done with an ion chamber with rings for uniformity check and radiochromic when needed.

Texas A\&M University (TAMU) [25] uses a K500 superconducting cyclotron (described above). The energy range is $8-70 \mathrm{MeV}$ tunable. The beam spot is $1^{\prime \prime}$ diameter. The dosimetry used is four/five scintillator array.

Tri-University Meson Facility (TRIUMF), located in Canada [32], utilizes a cyclotron to accelerate protons to energies between $65-120 \mathrm{MeV}$ on one beamline and $180-500 \mathrm{MeV}$ on another beamline. A 2- to 3-in square beam spot is available depending on the energy used. The dosimetry on low energy line is ion chambers calibrated against externally calibrated ion chamber. On the high-energy line dosimetry is a achieved with a combination of faraday cup, plastic scintillators, and PIN diodes; all agree very closely.

The Paul Scherrer Institute, Switzerland, Proton Accelerator Facility [33] can provide protons with energies between 60 and $300 \mathrm{MeV}$ in one facility and between 6 and $65 \mathrm{MeV}$ in another. Beam spot is $34 \mathrm{~mm}$ (low energy) and $9 \mathrm{~cm}$ (high energy) diameter. The dosimetry in the low energy facility is done using ion chambers or $\mathrm{CsI}(\mathrm{Tl})$ scintillators. In the high-energy side an ion chambers, PIN diodes and plastic scintillators are used.

Harvard University has a cyclotron that has been used extensively in the past for SEE testing. However, since the early 1990s this facility has decided to focus most of its resources on cancer treatment applications and research. Currently access to Harvard for SEE testing is very limited.

\section{Other SEE Test Facilities}

Although broad-beam accelerators are essential for SEE characterization, there are other approaches capable of providing information on SEEs. The limitations of broad-beam accelerator testing_-including limited availability, high cost, and lack of both detailed spatial and temporal information - have lead to the development of alternate approaches for measuring SEE sensitivity. They include pulsed lasers, ion microbeams, and $252 \mathrm{Cf}$.

A pulsed laser is a well-established tool for elucidating the spatial and temporal characteristics of SEEs [34]-[37]. The basic requirement is that the laser generate short $(\sim 1 \mathrm{ps})$ pulses of light, with pulse energies greater than a $\mathrm{nJ}$ and photon energies greater than the energy bandgap of the semiconductor. Added flexibility comes from being able to fire the laser repeatedly without damaging the device, from single shot to kilohertz rates, and to do so without the need for a vacuum. The light is typically focused to $\mathrm{a} \sim 1 \mu \mathrm{m}$ size spot and scanned across the device to obtain the spatial dependence of SEE sensitivity. The temporal characteristics of SEEs in dynamic circuits can be measured by synchronizing the circuit clock to the laser trigger and adding delay [38]. Although the charge generation mechanisms for ionizing particles differ fundamentally from those for ionizing photons, both experimental and theoretical investigations show that the resulting voltage transients are, in many cases, indistinguishable [39], [40]. The pulsed-laser technique does suffer from a significant limitation - the inability of the light to penetrate metal layers on the surface of a device. This is the reason why recent reports suggesting that a pulsed laser can be used to generate curves of SEE cross section versus LET are not promising [41]. One application of the pulsed laser that has, on occasion, proved invaluable is to ensure that both the devices selected for testing and the test equipment are functioning properly before being shipped to the accelerator facility.

A magnetically focused ion microbeam is a powerful tool for studying the basic mechanisms contributing to SEEs. The ion beam is generated by an accelerator and focused by a set of magnets to produce a beam with a diameter of $\sim 1 \mu \mathrm{m}$. One such facility, located at Sandia National Laboratory (SNL), generates ions with a maximum energy of $50 \mathrm{MeV}$. Of the ions available, those with ranges greater than $10 \mu \mathrm{m}$ typically have LETs less than $15 \mathrm{MeV}-\mathrm{cm}^{2} / \mathrm{mg}$. For testing, the devices are mounted in a vacuum chamber and the ion beam is either rastered across areas of interest or is fixed in one position. Rastering the beam permits the generation of detailed maps of the device response at specific locations around various sensitive transistors. In this way, images of both SEE sensitive areas and charge collection efficiency from specific junctions have been measured [42], [43]. Most of the SEE investigations using a microbeam have been reported by two groups-SNL in the U.S. and Gesellschaft fuer Schwerionenforschung (GSI) in Germany. Some of the technique's limitations are the short range of the ions available, the necessity of using a vacuum, limited number of ion LETs, and the damage induced by the ion beam in the device being tested.

The third approach involves using the decay products from a radioactive source, such as $252 \mathrm{Cf}$ to generate SEEs in circuits [44]-[46]. The source and the device are mounted close to each other in a vacuum chamber. The decay products fall into two energy ranges around average energies of 78 and $102 \mathrm{MeV}$. Therefore, not only are the ion energies and, consequently, the ranges relatively low, the uncertainty in energy leads to an uncertainty in both LET and range. The maximum available LET at normal incidence is $45 \mathrm{MeV}-\mathrm{cm}^{2} / \mathrm{mg}$. A major issue is the short range $(\sim 10 \mu \mathrm{m})$ of the ions that limits the usefulness of the technique, because devices with thick passivation layers or deep junctions cannot be tested reliably. Another issue is the radiation hazard for which stringent precautions must be taken to protect personnel. Nevertheless, for quick evaluations prior to doing accelerator testing, or for certain hardness assurance measurements, radioactive sources do offer a useful approach.

\section{ReVIEW of SEE RATE PREDICTIONS TeChNIQUES}

\section{A. Introduction}

Prediction of SEE rates involves a combination of experimental data, assumptions about the device, and knowledge of the energetic particle environment. This section discusses how the ground test data, as described above, can be used to predict rates for SEE due to energetic particles in a space environment. We summarize the current rate prediction techniques for heavy ions and protons, interspersed with a historical glimpse of the early evolution of the concepts and approaches. Table I lists 
TABLE I

Key Milestones in Development of SEE Rate Prediction Methods

\begin{tabular}{l|l|l|l}
\hline \multicolumn{1}{c|}{ Milestone } & \multicolumn{1}{c|}{ Date } & \multicolumn{1}{c|}{ Authors } & Reference \\
\hline First reported SEU in space & 1975 & Binder, Smith and Holman & [Bind1] \\
\hline $\begin{array}{l}\text { LET distribution concept is } \\
\text { introduced }\end{array}$ & 1977 & Heinrich & {$[$ Hein] } \\
\hline $\begin{array}{l}\text { First reported alpha particle upset } \\
\text { in ground-based ICs }\end{array}$ & 1979 & May and Woods & {$[$ May] } \\
\hline $\begin{array}{l}\text { Development of heavy ion SEU } \\
\text { rate prediction model based on } \\
\text { distributions of path length and } \\
\text { LET }\end{array}$ & 1978,1980 & Pickel and Blandford & {$[$ [Pick1], } \\
$\begin{array}{l}\text { First observations of proton- } \\
\text { induced SEU }\end{array}$ & 1979 & $\begin{array}{l}\text { Wyatt, McNulty, Toumbas, } \\
\text { Rothwell and Filz }\end{array}$ & [Wyat] \\
$\begin{array}{l}\text { Development of semi-empirical } \\
\text { model for proton SEU rate }\end{array}$ & 1983 & Guenzer, Wolicki and Allas & [Guen] \\
$\begin{array}{l}\text { CREME suite of codes combine } \\
\text { environment and rate prediction } \\
\text { tools in standardized package }\end{array}$ & 1986 & Bendel and Petersen & [Bend] \\
$\begin{array}{l}\text { Development of Effective Flux } \\
\text { approach for heavy ion SEU rate }\end{array}$ & 1988 & Binder & {$[$ Adam] } \\
\hline
\end{tabular}

the key milestones for development of SEE rate prediction techniques. Much work has been done to refine the early methods in the ensuing years, and the reader is referred to the TNS and several sets of Nuclear and Space Radiation Effects Conference (NSREC) Short Course Notes [47]-[49] to follow that trail. See the Short Course notes by Petersen [47] for an excellent review of the historical evolution of the development and use of rate prediction concepts.

SEEs are related to charge generation along the path of a primary or secondary ionizing particle, charge collection on circuit nodes, and circuit response to the charge transient. Both the total collected charge and the rate of charge collection can be important to triggering the effect. SEE rate prediction models typically use ground test data to extract information about the device sensitivity, measured in terms of cross section (CS) and critical charge (Qc), as a function of LET and/or proton energy. Testing methods have been devised to generate this information, as described in the previous sections. Once the CS versus LET or CS versus proton energy data have been experimentally acquired, there are established techniques for using the data to predict SEE rates in a given space environment. The rate prediction methods do a fairly good job of predicting what is actually seen on-orbit. Of course, the quality of the predictions is a function of the quality of the test data and the skill of the modeler, taking into account the assumptions and limitations of the models.

\section{B. Heavy Ion Predictions}

To first order, the linear energy deposition rate $(\mathrm{MeV} / \mu \mathrm{m})$ drives the effects. This allows simplification of the prediction problem through use of energy transfer (LET) spectra, as first developed by Heinrich [5]. All the ion types and distributions of energy in the space environment can be reduced to their LET, and deposited energy can be estimated as LET times the chordlength through the sensitive volume. With this simplification, the problem to be solved is to identify the size of the sensitive volume, calculate the rate of ion hits and the consequent energy depositions, and determine the subset of total ion hits that cause SEE.

The SEE rate is the product of the sensitive area on the chip and the flux of ions in the environment that can cause upset when they hit the sensitive area. The threshold for the effect determines the effective flux. The problem is complicated by the angular dependence since the amount of energy deposited in the sensitive volume depends on chordlength, which in turn depends on angle of incidence. The modeling problem can be approached from two directions: from a microscopic viewpoint (the chordlength approach) or from a macroscopic viewpoint (the effective flux approach). Both approaches give similar results and are effectively equivalent if the same geometric assumptions are made. The chordlength model determines the minimum charge required for upset from cross section versus LET test data, considers the distribution of LET in the environment, and puts a criterion on each ion interaction with a sensitive volume to select a set of ions (and associated flux) that exceed the minimum charge. The effective flux model transforms the ion flux in the space environment to an effective flux (capable of causing SEE) based on measured cross section versus LET test data for the chip. Several rate prediction methodologies and codes are discussed in the literature, but they all fall into one of these two general categories.

1) Chordlength model: The original Chordlength model was introduced by Pickel and Blandford in 1978 [4] and developed into a computer code (CRIER) in 1980 [50]. The sensitive volume is modeled as a rectangular parallelepiped (RPP) with lateral dimensions $x$ and $y$ and thickness $z$. The saturation cross section per bit $\left(\mathrm{CS}_{m}\right)$ is given by the product of $x$ and $y$; conversely $x$ and $y$ are determined by measurement of $\mathrm{CS}_{m}$, taking into account the number of bits in the chip. The RPP approximates the depletion region beneath a p-n junction that is determined to be a sensitive volume. The ion is assumed 
to travel in a straight line and the path through the RPP is $S$, determined by thickness, $z$, and the angle of incidence, $\theta$. Ion plasma track structure is ignored. Charge is also allowed to be collected along a funneling distance $S_{f}$ that adds to the chordlength $\mathrm{S}$ through the depletion region. Epitaxial layer thickness may limit charge collection by funneling. The energy deposited in the sensitive volume from an ion with LET $L$ is

$$
\mathrm{E}=\left(\mathrm{S}+\mathrm{S}_{f}\right) \mathrm{L}
$$

This energy is converted to charge in accordance with the ionization energy (3.6 eV/carrier pair for silicon) and it is assumed that all charge that is generated within the charge collection length $\mathrm{S}+\mathrm{S}_{f}$ is collected by the circuit node. It is assumed that there is a sharp threshold for upset-ion hits below a threshold LET do not cause upset, hits above the threshold cause upset. The classic RPP method utilizes an integral LET distribution and an analytic differential chordlength distribution function $f(S$ and integrates over the chordlengths through the $\mathrm{RPP}$. The rate is expressed as

$$
\begin{aligned}
\mathrm{R}(\mathrm{Ec}) & =\mathrm{A}_{\mathrm{p}} \int \Phi[\operatorname{Lt}(\mathrm{S}, \mathrm{Ec})] \mathrm{f}(\mathrm{S}) \mathrm{dS} \\
\mathrm{S}_{\min } & =0 \\
\mathrm{~s}_{\max } & =\left(\mathrm{x}^{2}+\mathrm{y}^{2}+\mathrm{z}^{2}\right)^{\frac{1}{2}}
\end{aligned}
$$

where the limits on the integral are from zero to the maximum pathlength through the RPP, $A_{p}$ is average projected area of the $\mathrm{RPP}, \Phi(\mathrm{L})$ is integral flux, $\mathrm{E}_{c}$ is the threshold energy for generating $\mathrm{Q}_{c}$, and $\mathrm{L}_{t}\left(\mathrm{~S}, \mathrm{E}_{c}\right)$ is the minimum LET which depends on chordlength through

$$
\mathrm{L}_{t}\left(\mathrm{~S}, \mathrm{E}_{c}\right)=\frac{\mathrm{E}_{c}}{\left(\mathrm{~S}+\mathrm{S}_{f}\right)}
$$

where the chord length random variable $S$ has been modified to account for charge collection by funneling and $\mathrm{E}_{\mathrm{c}}$ is defined by the critical charge. Inputs to the classic model are $x, y, z, \mathrm{~S}_{f}$, and $\mathrm{E}_{c}$.

Alternative formulations that use an integral chordlength and differential LET distribution were introduced by Petersen and Shapiro in 1982 [51]. The two approaches are fundamentally equivalent. They differ in how they handle the complexities of integrating over discontinuities.

The classic RPP model assumes a step function for the cross section versus LET curve. However, most devices exhibit a gradual rise from threshold to saturation because chip response generally is the composite of multiple types of sensitive volumes with different thresholds and with distributions on their parameters. Petersen was the first to address this issue, suggesting that the cross-section curve be divided into several steps in order to more accurately represent it [52]. The common approach is to weigh $\mathrm{R}(\mathrm{E})$ with the normalized experimental cross-section data

$$
\mathrm{R}=\int \mathrm{R}(\mathrm{E}) f(\mathrm{E}) \mathrm{dE}
$$

where the integration range is from the measured threshold $\mathrm{E}_{c}$ to the measured value at saturation $\mathrm{E}_{\mathrm{sat}}$, and $f(\mathrm{E})$ is the cross section versus LET curve converted to a probability density, often described by the four parameter Weibull distribution. The function is the rate at which an energy of $\mathrm{E}$ or greater is deposited in the sensitive volume. Moreover, $f(\mathrm{E})$ may be regarded as the probability density for an event caused by deposition of $\mathrm{E}$ or greater. Thus the integral is the expectation of $\mathrm{R}(\mathrm{E})$ with the probability $f(\mathrm{E})$. This approach is commonly called the Integral RPP (IRPP) model.

The integral in (2) is solved numerically. The original implementation was in the CRIER code [50] and a version of this code is implemented in CREME suite of codes [53] and also in commercial codes. The integral in (4) is solved by dividing the data set into a number of bins based on LET. The data set is divided into a number of bins based on LET and the integral in (4) is also solved numerically.

2) Effective Flux Model: The original Effective Flux model was introduced by Binder in 1988 [54]. The method is based on consideration of the range of incident angles that can produce an SEU and the ion flux contained in that range. The model assumes an isotropic flux as a function of LET, F(L), incident on a thin lamina. If the threshold for upset is $\mathrm{L}_{t}$ and $\mathrm{L}>\mathrm{L}_{t}$, then all incident angles produce upset. If $\mathrm{L}<\mathrm{L}_{t}$, there is a critical angle, $\theta_{c}$, which produces upset, where

$$
\cos \left(\theta_{c}\right)=\frac{\mathrm{L}}{\mathrm{L}_{t}}
$$

The ion flux in the environment $\Phi$ (L) can be transformed to an effective flux $\Phi_{e}(\mathrm{~L})$ for an assumed cutoff angle $\theta_{\mathrm{c}}$. The effective flux is sometimes called redistributed flux. Then the rate is calculated by

$$
\mathrm{R}=\int \Phi_{e}\left(\mathrm{~L}_{t}\right) \mathrm{d} \mathrm{CS}(\mathrm{L})
$$

where $\mathrm{CS}(\mathrm{L})$ is the measured cross section versus LET test data and the limits on the integral are from zero to the maximum LET in the environment.

In general, the effective flux model predictions agree with the chordlength model predictions when the effective flux calculation is performed allowing for the appropriate geometry. This agreement is not surprising if the thickness of the sensitive volume is small $(<10 \%)$ compared to the lateral dimensions.

3) Figure of Merit Approximation: A Figure of Merit approximation for heavy ion SEU was introduced by Petersen in 1983 [52] before wide distribution of codes for the more exact methods, and further refined in 1998 [55]. The Petersen approximation equation is

$$
\mathrm{R}=\frac{\mathrm{K} \mathrm{CS}_{m}}{\mathrm{~L}_{t}^{2}}
$$

where $\mathrm{R}$ is rate in upsets/bit-day, $\mathrm{CS}_{m}$ is saturation cross section in $\mathrm{cm}^{2}, \mathrm{~L}_{t}$ is the LET at $25 \%$ of $\mathrm{CS}_{m}$ in $\mathrm{MeV}-\mathrm{cm}^{2} / \mathrm{mg}$, and $\mathrm{K}$ is a constant determined by the environment. The value of $K$ depends on device sensitivity, ranging from approximately 95 for hard devices to 360 for soft devices [55]. The equation was originally developed for quick device comparisons for heavy ion upset. The Figure of Merit approach has also been applied to proton-induced upset and has been studied for predicting heavy ion upset from proton data or proton upset from heavy ion data [55]. 


\section{Proton Predictions}

From the device perspective, the fundamental upset mechanism is the same for heavy ions and protons. The difference is whether a primary heavy ion causes upset, or a secondary ion recoil from a nuclear interaction of a proton within the device causes the upset. Rate prediction for proton nuclear-reaction-induced SEU is simplified by the isotropic nature of nuclear reaction cross sections, which removes most of the angular dependence from the SEU mechanism. Proton upset rates are driven by nuclear reactions of the protons with the semiconductor material and determination of the recoil energy spectra is key to the solution. Rate prediction approaches have taken two general approaches: analytical calculations that consider the details of proton-induced nuclear reactions and the secondary ions, and semi-empirical approaches that rely on measured chip response to protons. The majority of proton upset rate calculations have used the semi-empirical approach.

1) Bendel Model: The original approach for predicting proton-induced SEU was developed by Bendel and Petersen and came from the observation that much of the proton SEU cross-section data as a function of proton energy follows a relationship resembling the proton nuclear reaction cross section in silicon [56]. The general Bendel two-parameter model has the form

$$
\sigma_{p}=\sigma_{m}\left[1-\exp \left(-0.18 \mathrm{Y}^{0.5}\right)\right]^{4}
$$

where $\sigma_{p}(\mathrm{E})$ is a cross section at energy $\mathrm{E}$ in units of $10^{12}$ upsets per proton $/ \mathrm{cm}^{2}$ per bit, $\sigma_{m}$ is the maximum upset cross section, and

$$
\mathrm{Y}=\left(\frac{18}{\mathrm{~A}}\right)^{0.5}(\mathrm{E}-\mathrm{A})
$$

where $\mathrm{E}$ and $\mathrm{A}$ are in megaelectronvolts.

The original formulation had both a threshold and a limiting cross section but observed that a single parameter was adequate to describe the data available at the time. The Bendel parameter A was introduced on a semi-empirical basis as the proton incident energy threshold for proton reactions that cause upsets. The model has more uncertainty for low energy protons [56].

As more data became available, it became clear that the response of some modern smaller feature size devices was better modeled with the use of both threshold and cross-section parameters. An improved two-parameter Bendel model was suggested by two groups at about the same time and this is the form that currently has the widest acceptance [57], [58]. The two-parameter model is expressed as

$$
\sigma_{p}=\left(\frac{\mathrm{B}}{\mathrm{A}}\right)^{14}\left[1-\exp \left(-0.18 \mathrm{Y}^{0.5}\right)\right]^{4}
$$

where $\mathrm{A}$ and $\mathrm{B}$ are empirically determined constants unique to a device and $\mathrm{Y}$ is defined in (9).

The parameter $\mathrm{A}$ is related to the apparent upset energy threshold, while the ratio $(\mathrm{B} / \mathrm{A})^{14}$ is associated with the saturation cross section observed at high energies. Note that the one-parameter model has B fixed at a value of 24. The advantage of the two-parameter model is that it allows better fitting of the experimental data in the high energy regions, particularly for small geometry devices, while preserving the apparent low energy proton upset threshold.

The average upset rate for a given orbit is determined by integration of the cross-section curve defined by (10) over the orbitintegrated proton energy spectrum at the device. The empirical parameters are determined from experimental data by measuring the proton upset cross section at one or more proton energies. With sufficient experimental data, other curve fits (e.g., Weibull) besides (10) can be used to calculate the rate.

Note that the proton upset rate depends on the probability of a nuclear interaction in the device and pathlength of the proton in the device is not a factor. This is in contrast to the case of heavy ion upset where the rate depends on the total energy deposition and the path-length must be taken into consideration.

\section{EXAMPLES OF BREAKDOWN OF MODELS AND TEST METHODS}

\section{A. Introduction}

While the test methods and rate prediction approached described above are very robust and have been proven time and time again to sufficiently estimate the observed on-orbit SEE rate (see, for example, [59]), these methods do have limitations; some of these limitations are discussed in [59]. In this section, we summarize several observed effects that either forced researches to modify the existing test methods and/or rate prediction approaches or are current topics of ongoing research. This list is obviously not inclusive but is broad enough to convince the reader that care must be taken whenever evaluating a new technology for applications in the space radiation environment.

\section{B. Charge Collection by Diffusion}

The classical upset models assume that the charge is collected in times much shorter than device response time. In reality, charge is collected promptly (picoseconds) from high-field regions such a depletion regions and in charge funneling regions, but relatively slowly (nanoseconds to microseconds) by diffusion from low-field regions such as substrates. If the mechanism for the effect has characteristic times on the time for charge collection by diffusion, then the slower charge collection time needs to be considered. Many DRAMs and SRAMs have such long time constants. Another good example of this effect can be seen in the mechanism for single-event latchup (SEL) [60]. The cross sections for upsets is radically different for diffusion dominated devices as compared to drift dominated devices. The cross section for large LET ions is greater than the dimensions of drift regions and increases as the LET of the ions increases. Numerical analysis of the problem is still a field of current research.

\section{Bipolar Effects in SOI}

At first glance, Silicon-On-Insulator (SOI) would seem to be much less sensitive to upset since the pathlengths are small and the amount of charge that can be deposited by an ion is limited. However, it has been observed that SOI often upsets at unexpectedly lower LET thresholds. The reason is that there is a parasitic bipolar amplification of the charge generated by the ion. 
The upset rate models can be augmented to account for this extra charge source [61].

\section{Thick Sensitive Volumes}

The concept of effective LET is based on the fact that the sensitive volumes are thin RPPs. However, not all devices have thin sensitive volumes (some are not RPPs at all). When the heavy ion beam is rotated at some off normal angle, the ions have a pathlength distribution that is much more complicated than simple cosine law, i.e., effective LET concept is not valid in thick sensitive volumes. This is due to the ions that pass through the edge of the sensitive volume, known as edge effects. One approach to dealing with edge effects is given in [62 ] and the references therein.

\section{E. Ion Track Structure Effects}

The current rate prediction models assume that the line of charge created as the ion passes through the material is very thin compare to the collection volume dimensions. It has been speculated that as technology scales to smaller dimensions that the lateral spread in the line of charge due to delta rays will become important. The exact scale where this will become an issue is highly disputed. Limited data showing a slight dependence on track structure has been presented in [63], [64]. An extensive study on selected modern technologies using high and low energy ions with the same LET (track radius is different for ions with different energies) was presented in [65]; no effect was observed for these devices.

\section{F. SETs in Optocouplers and Optical Data Links}

High-speed data links, including Optocouplers, can be sensitive to proton induced SEEs [66]-[69]. In [66], the authors report that the proton-induced SET cross section for a optical data links depends on angle of incidence of the proton beam, a result that differs from the classical view of proton testing. In that paper, the angle dependence was determined to be due to direct ionization effects due to protons. A similar effect has been noted in optocouplers [63], [67], [69]. The cross section for these devices depends on the superposition of the cross section due to direct ionization effects with that for indirect ionization effects. While several authors have attempted to develop on-orbit SET rate prediction approaches, there is no currently accepted rate prediction approach for this effect that is cost effective to implement. Most either require large amounts of data to be taken at various angles and proton energies or rely on computer codes that do not accurately model the effects.

\section{G. Charge Collection in SOI}

For certain SOI devices the silicon volume is the area under the gate plus some region extending into the drain and source [70]. Also, it is possible to have charge collection from below the buried oxide in SOI technologies that have very thin buried oxide layers [70], [71].

From this, it is easy to see that the sensitive volume is not a simple RPP defined by the volume under the gate, but a complex structure that depends on variations in the buried oxide thickness, ion energy, LET and range, and other factors. There is no current rate prediction method that will accurately model this effect.

\section{H. Proton-Induced SEUs in Sensitive Volumes With Large Aspect Ratios}

In [72], it was shown that the proton-induce SEU cross section can depend on the proton beam angle of incidence for SOI (including SOS) devices that have sensitive volumes with one dimension that is much longer than the others and have a sufficiently large critical charge. The authors also show that the angular effect has an energy dependence. The angular effects observed experimentally to date have been described as being a result of the device response to the angle distribution of the recoil atoms produced from nuclear spallation reactions [72], [73].

Recall that one of the basic assumption for using the Bendel model (one or two parameter) is that the cross section is independent of the proton beam angle of incidence. The Bendel approach could underpredict the SEU rate; Monte Carlo codes have been used to model this effect [72], [73] but will need further development to be useful as a rate prediction tool.

\section{CONCLUSION}

Over the past 27 years, SEE rate prediction approaches have been developed based on observed on-orbit data and ground-based research. The Cosmic Ray Research Satellite (CRRES) [74], circa 1980 (no longer active), and Microelectronics and Photonics Test Bed (MPTB) [75], circa 1998 (still active), are two major spaceflight instruments that contain SEE experiments. These experiments have been vital to the validation of the traditional SEE rate prediction approaches, as well as other radiation effects research. However, there are no flight data on technologies that are less than a decade and a half old to show that there is a comprehensive understanding of how to use the existing models and test methods on current technology. Future spaceflight opportunities, like the Living With a Star's Space Environment Testbed [76], are absolutely necessary to ensure that accurate rate prediction models and test methods are being used.

The key result of the SEE research over the past three decades has been to develop fairly well-defined test approaches, rate prediction methods and test facilities that incorporate knowledge of the space radiation environment. These methods are valid for a subset of the technologies that are available to today's spacecraft designers. Further development of each of these areas will be needed to maintain a core competency level that allows safe reliable space systems to be launched in the future.

\section{ACKNOWLEDGMENT}

The authors would like to thank R. Ladbury, T. Oldham, B. Fodness, and C. Poivey for technical discussions and review of this work. They would also like to thank D. Cochran for support during the preparation of the paper. 


\section{REFERENCES}

[1] P. E. Dodd and L. W. Massengill, "Basic mechanisms and modeling of single-event upset in digital microelectronics," IEEE Trans. Nucl. Sci., vol. 50, pp. -, June 2003.

[2] J. T. Wallmark and S. M. Marcus, "Minimum size and maximum packing density of nonredundant semiconductor devices," IRE Proc., vol. 50, p. 286, 1962.

[3] D. Binder, E. C. Smith, and A. B. Holman, "Satellite anomalies from galactic cosmic rays," IEEE Trans. Nucl. Sci., vol. NS-22, p. 2675, 1975.

[4] J. C. Pickel and J. T. Blandford Jr., "Cosmic ray induced errors in MOS memory cells," IEEE Trans. Nucl. Sci., vol. NS-25, p. 1166, 1978.

[5] W. Heinrich, "Calculation of LET spectra of heavy cosmic ray nuclei at various absorber depths," Radiation Effects, vol. 34, p. 143, 1977.

[6] T. C. May and M. H. Woods, "Alpha particle induced soft errors in dynamic RAM memories," IEEE Trans. Electron. Devices, vol. ED-26, p. 2, 1979.

[7] R. C. Wyatt, P. J. McNulty, P. Toumbas, P. L. Rothwell, and R. C. Filz, "Soft errors induced by energetic protons," IEEE Trans. Nucl. Sci., vol. NS-26, p. 4905, 1979

[8] C. S. Guenzer, E. A. Wolicki, and R. G. Allas, "Single event upset of dynamic RAM's by neutrons and protons," IEEE Trans. Nucl. Sci., vol. NS-26, p. 5048, 1979.

[9] W. A. Kolasinski, J. B. Blake, J. K. Anthony, W. E. Price, and E. C. Smith, "Simulation of cosmic-ray induced soft errors and latchup in integrated-circuit computer memories," IEEE Trans. Nucl. Sci., vol. NS-26, p. 5087,1979

[10] E. G. Stassinopoulos, "Radiation environment of space," in Proc. 1990 Nuclear and Space Radiation Effects Conf. Short Course, Reno, NV.

[11] J. Barth, "Modeling space radiation environments," in Proc. $1997 \mathrm{Nu}$ clear and Space Radiation Effects Conf. Short Course, Snowmass, CO, July 27, 1997.

[12] C. Dyer, "Space radiation dosimetry," in Proc. 1998 Nuclear and Space Radiation Effects Conf. Short Course, Newport Beach, Ca..

[13] J. Mazur, "The radiation environment inside and outside the spacecraft," in Proc. 2002 Nuclear and Space Radiation Effects Conference Short Course, Phoenix, Az.

[14] J. L. Barth, C. S. Dyer, and E. G. Stassinopoulos, "Space, atmospheric, and terrestrial radiation environments," IEEE Trans. Nucl. Sci., vol. 50, pp. -, June 2003.

[15] E. L. Petersen, "Cross section measurements and upset rate calculations," IEEE Trans. Nucl. Sci., vol. 43, pp. 2805-2813, 1996.

[16] R. Koga, W. A. Kolasinski, M. T. Marra, and W. A. Hanna, "Techniques of microprocessor testing and SEU rate prediction," IEEE Trans. Nucl. Sci., vol. NS-32, pp. 4219-4224, 1985.

[17] T. L. Turflinger, M. V. Davey, and B. M. Mappes, "Single event effects in analog-to-digital converters: Device performance and system impact," IEEE Trans. Nucl. Sci., vol. 41, pp. 2187-2194, 1994.

[18] R. Katz, K. LaBel, J. J. Wang, B. Cronquist, R. Koga, S. Penzin, and G. Swift, "Radiation effects on current field programmable technologies," IEEE Trans. Nucl. Sci., vol. 44, pp. 1945-1956, 1997.

[19] J. L. Titus, C. F. Wheatley, D. I. Burton, I. Mouret, M. Allenspach, J. Brews, R. Schrimpf, K. Galloway, and R. L. Pease, "Impact of oxide thickness on SEGR failure in vertical power MOSFETs: Development of a semi-empirical expression," IEEE Trans. Nucl. Sci., vol. 42, pp. 1928-1934, 1995.

[20] M. Allenspach, I. Mouret, J. L. Titus, C. F. Wheatley, R. L. Pease, J. R. Brews, R. D. Schrimpf, and K. F. Galloway, "Single event gate rupture in power MOSFETs: Prediction of breakdown biases and evaluation of oxide thickness dependence," IEEE Trans. Nucl. Sci., vol. 42 , pp. 1922-1927, 1995.

[21] Tandem Van de Graaff Facility, SEU Test Facility: User Guide. Upton, NY: Brookhaven Nat. Lab., 1991.

[22] The 88-Inch Cyclotron, World Wide Web Page (2002, Jan.) http://www.lbl.gov/nsd/user88/88description.html [Online]

[23] Aerospace Single Event Effects Testing, World Wide Web Page (2001, Aug. 14). http://www.aero.org/seet/index.html [Online]

[24] Radiation Effects Facility - Cyclotron Institute, World Wide Web Page (2002, Jan. 3). http://cyclotron.tamu.edu/ref/ [Online]

[25] Cyclotron Institute, World Wide Web Page (2001, Oct. 29). http://cyclotron.tamu.edu/index.html [Online]

[26] T. L. Grimm, C. C. Compton, D. Lawton, F. Marti, X. Y. Wu, and R. C. York, "Design Study of a Single Event Effects Test Facility at the NSCL," Goddard Space Flight Center, Greenbelt, MD, 1999.

[27] Cyclotron Research Centre World Wide Web Pagehttp://www. cyc.ucl.ac.be/ [Online]
[28] l'Institut de Physique Nucléaire, World Wide Web Pagehttp://ipnweb in $2 p 3 . f r$ [Online]

[29] Grand Accelerateur National D'Ions Lourds, World Wide Web pagehttp://www. ganil.fr/ [Online]

[30] Crocker Nuclear Lab., World Wide Web Pagehttp://crocker. ucdavis.edu [Online]

[31] Indiana Univ. Cyclotron Facility, World Wide Web Pagehttp://www iucf.indiana.edu/ [Online]

[32] Tri-University Meson Facility, World Wide Web Pagehttp://www. triumf.ca/homepage.html [Online]

[33] Paul Scherrer Institute, World Wide Web Pagehttp://www. psi.ch/index_e_proton.shtml [Online]

[34] S. Buchner, D. Wilson, K. Kang, D. Gill, J. Mazur, W. D. Raburn, A. B. Campbell, and A. R. Knudson, "Laser simulation of single event upsets," IEEE Trans. Nucl. Sci, vol. NS-34, pp. 1228-1233, Dec 1987.

[35] S. C. Moss, S. D. LaLumondiere, J. R. Scarpulla, K. P. MacWilliams, W. R. Crain, and R. Koga, "Correlation of picosecond laser-induced latchup and energetic particle-induced latchup in CMOS test structures," IEEE Trans. Nucl. Sci, vol. 42, pp. 1948-1956, Dec. 1995.

[36] V. Pouget, H. Lapuyade, P. Fouillat, D. Lewis, and S. Buchner, "Theoretical investigation on an equivalent laser LET," Microelectronics Reliability, vol. 41, p. 1513, 2001.

[37] A. M. Chugg, R. Jones, M. Moutrie, C. S. Dyer, C. Sanderson, and A. Wraight, "Probing the charge-collection sensitivity profile using a picosecond pulsed laser at a range of wavelengths," IEEE Trans. Nucl. Sci, vol. 49, pp. 2969-2976, 2002.

[38] R. Schneiderwind, D. Krening, S. Buchner, K. Kang, and T. R. Weatherford, "Laser confirmation of SEU experiments in GaAs MESFET combinatorial logic," IEEE Trans. Nucl. Sci., vol. 39, pp. 1665-1672, 1992.

[39] D. McMorrow, T. R. Weatherford, A. R. Knudson, L. H. Tran, J. S. Melinger, and A. B. Campbell, "Single event dynamics of high-performance HBT's and GaAs MESFET's," IEEE Trans. Nucl. Sci., vol. 40, pp. 1858-1866, 1993

[40] R. L. Pease, A. L. Sternberg, Y. Boulghassoul, L. W. Massengill, S. Buchner, D. McMorrow, D. S. Walsh, G. L. Hash, S. D. LaLumondiere, and S. C. Moss, "Comparison of SET's in bipolar linear circuits generated with an ion microbeam, laser light, and circuit simulation," IEEE Trans. Nucl. Sci., vol. 49, pp. 3163-3170, 2002.

[41] R. Jones, A. M. Chugg, C. M. S. Jones, P. H. Duncan, C. S. Dyer, and C. Sanderson, "Comparison between SRAM SEE cross-sections from ion beam testing with those obtained using a new picosecond pulsed laser facility," IEEE Trans. Nucl. Sci., vol. 47, pp. 539-544, 2000.

[42] F. W. Sexton, "Microbeam studies of single-event effects," IEEE Trans. Nucl. Sci., vol. 43, pp. 687-704, 1996.

[43] S. Metzger, J. Dreute, W. Heinrich, J. Roecher, B. E. Fischer, R. HarboeSorenson, and L. Adams, "Heavy ion microscopy of single-event upsets in CMOS SRAM's," IEEE Trans. Nucl. Sci., vol. 41, pp. 589-592, 1994

[44] J. T. Blandford and J. C. Pickel, "Use of Cf-252 to determine parameters for SEU rate calculations," IEEE Trans. Nucl. Sci., vol. NS-32, pp. 4282-4287, 1985

[45] D. Mapper, T. K. Sanderson, J. H. Stephen, J. Farren, L. Adams, and R Horboe-Sorenson, "An experimental study of the effect of absorbers on the LET of the fission particles emitted by Cf-252," IEEE Trans. Nucl. Sci., vol. NS-32, pp. 4276-4281, 1985.

[46] A. Costantine, J. W. Howard, M. Becker, R. C. Block, L. S. Smith, G A. Soli, and M. C. Stauber, "A new method for using ${ }^{252} \mathrm{Cf}$ in SEU testing," IEEE Trans. Nucl. Sci., vol. 37, pp. 1916-1921, 1990.

[47] E. Petersen, "Single event analysis and prediction," Proc. IEEE NSREC Short Course, 1997.

[48] L. Massengill, "SEE modeling and prediction techniques," Proc. IEEE NSREC Short Course, 1993.

[49] J. C. Pickel, "Single event upset mechanisms and predictions," Proc. IEEE NSREC Short Course, 1983.

[50] J. C. Pickel and J. T. Blandford Jr., "Cosmic ray induced errors in MOS devices,” IEEE Trans. Nucl. Sci., vol. NS-27, p. 1006, 1980.

[51] E. L. Petersen, P. Shapiro, J. H. Adams Jr., and E. A. Burke, "Calculation of cosmic ray induced soft upsets and scaling in VLSI devices," IEEE Trans. Nucl. Sci., vol. NS-29, p. 2055, 1982.

[52] E. L. Petersen, J. B. Langworthy, and S. E. Diehl, "Suggested single event upset figure of merit," IEEE Trans. Nucl. Sci., vol. NS-30, p. 4533, 1983.

[53] J. H. Adams Jr., "Cosmic Ray Effects on Microelectronics, Part IV,", NRL Memo. Rep. 5901, 1986.

[54] D. Binder, "Analytic SEU rate calculations compared to space data," IEEE Trans. Nucl. Sci., vol. 35, p. 1570, 1988.

[55] E. L. Petersen, "The SEU figure of merit and proton upset rate calculations," IEEE Trans. Nucl. Sci., vol. 45, p. 2550, 1998. 
[56] W. L. Bendel and E. L. Petersen, "Proton upsets in orbit," IEEE Trans. Nucl. Sci., vol. NS-30, p. 4481, 1983.

[57] Y. Shimano, T. Goka, S. Kuboyama, K. Kawachi, T. Kanai, and Y. Takami, "The measurement and prediction of proton upset," IEEE Trans. Nucl. Sci., vol. 36, p. 2344, 1989.

[58] W. J. Stapor, J. P. Meyers, J. B. Langworthy, and E. L. Petersen, "Two parameter Bendel model calculations for predicting proton induced upset," IEEE Trans. Nucl. Sci., vol. 37, p. 1966, 1990.

[59] E. L. Petersen, "Predictions and observations of SEU rates in space," IEEE Trans. Nucl. Sci., vol. 44, p. 2174, 1997.

[60] A. H. Johnston, "Latchup in CMOS from single particles," IEEE Trans. Nucl. Sci., vol. 37, p. 1866, 1990.

[61] S. E. Kerns, L. W. Massengill, D. V. Kerns, M. L. Alles, T. W. Houston, H. Lu, and L. R. Hite, "Model for CMOS/SOI single event vulnerability," IEEE Trans. Nucl. Sci., vol. 36, p. 2305, 1989.

[62] L. W. Connel, P. J. McDaniel, A. K. Prinja, and F. W. Sexton, "Modeling the heavy ion upset cross section," IEEE Trans. Nucl. Sci., vol. 42, pp. 73-82, 1995

[63] W. J. Stapor, P. T. McDonald, A. R. Knudson, A. B. Campbell, and B. G. Glagola, "Charge collection in silicon for ions of different energy but same linear energy transfer (LET)," IEEE Trans. Nucl. Sci., vol. 35, p. $1585,1988$.

[64] L. W. Connell, F. W. Sexton, P. J. McDaniel, and A. K. Prinja, "Modeling the heavy ion cross-section for single event upset with track structure effects: The HIC-UP-TS model," IEEE Trans. Nucl. Sci., vol. 43, p. 2814, 1996.

[65] P. E. Dodd, O. Musseau, M. R. Shaneyfelt, F. W. Sexton, C. D'hose, G. L. Hash, M. Martinez, R. A. Loemker, J.-L. Leray, and P. S. Winokur, "29 Impact of ion energy on single-event upset," IEEE Trans. Nucl. Sci., vol. 45, p. 2483, 1998.

[66] P. W. Marshall, C. J. Dale, M. A. Carts, and K. A. LaBel, "Particle induced bit errors in high performance data links for satellite data management," IEEE Trans. Nucl. Sci., vol. 41, pp. 1958-1965, Dec. 1994.

[67] K. A. LaBel, P. W. Marshall, C. J. Marshall, M. D'Ordine, M. A. Carts, G. Lum, H. S. Kim, C. M. Seidleck, T. Powell, R. Abbott, J. L. Barth, and E. G. Stassinopoulos, "Proton-induced transients in optocouplers: In-flight anomalies, ground irradiation test, mitigation and implications," IEEE Trans. Nucl. Sci, vol. 44, pp. 1885-1892, 1997.
[68] R. A. Reed, P. W. Marshall, A. H. Johnston, J. L. Barth, C. J. Marshall, K. A. LaBel, M. D'Ordine, H. S. Kim, and M. A. Carts, "Emerging optocoupler issues with energetic particle-induced transients and permanent radiation degradation," IEEE Trans. Nucl. Sci., vol. 45, pp. 2833-2841, 1998.

[69] A. H. Johnston, T. Miyahara, G. M. Swift, S. M. Guertin, and L. D. Edmonds, "Angular and energy dependence of proton upset in optocouplers," IEEE Trans. Nucl. Sci., vol. 46, pp. 1335-1341, 1999.

[70] P. E. Dodd, M. R. Shaneyfelt, K. M. Horn, D. S. Walsh, G. L. Hash, T. A. Hill, B. L. Draper, J. R. Schwank, F. W. Sexton, and P. S. Winokur, "SEU-sensitive volumes in bulk and SOI SRAM's from first-principles calculations and experiments," IEEE Trans. Nucl. Sci., vol. 48, pp. 1893-1903, 2001.

[71] J. R. Schwank, P. E. Dodd, M. R. Shaneyfelt, G. Vizkelethy, B. L. Draper, T. A. Hill, D. S. Walsh, G. L. Hash, B. L. Doyle, and F. D. McDaniel, "Charge collection in SOI capacitors and circuits and its effect on SEU hardness," IEEE Trans. Nucl. Sci., vol. 49, pp. 2937-2947, 2002.

[72] R. A. Reed, P. W. Marshall, H. Kim, P. J. McNulty, B. Fodness, T. Jordan, R. Reedy, C. Tabbert, M. (S. T.) Liu, W. Heikkila, S. Buchner, R. Ladbury, and K. A. LaBel, "Evidence for angular effects in proton-induced single-event upsets," IEEE Trans. Nucl. Sci., vol. 49, pp. 3038-3044, 2002.

[73] R. A. Reed, P. J. McNulty, and W. G. Abdel-Kader, "Implications of angle of incidence in SEU testing of modern circuits," IEEE Trans. Nucl. Sci., vol. 41, pp. 2049-2054, 1994.

[74] A. B. Campbell, "SEU flight data from the CRRES MEP," IEEE Trans. Nucl. Sci., vol. 38, pp. 1647-1654, 1991.

[75] A. Campbell, S. Buchner, E. Petersen, B. Blake, J. Mazur, and C. Dyer, "SEU measurements and predictions on MPTB for a large energetic solar particle event," IEEE Trans. Nucl. Sci, vol. 49, pp. 1340-1344, 2002.

[76] LWS SET World Side Web Page http://lws-set.gsfc.nasa.gov/ [Online] 